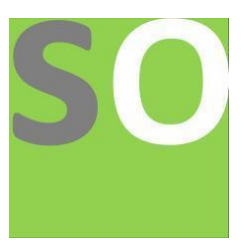

Article title: Superluminal velocity beyond the scope of application of relativity

Authors: Chang-Wei Hu[1]

Affiliations: FederaBeijing Relativity Theory Researchtion, Beijing, China[1]

Orcid ids: 0000-0001-6218-208X[1]

Contact e-mail: huchangwei@sina.cn

License information: This work has been published open access under Creative Commons Attribution License http://creativecommons.org/licenses/by/4.0/, which permits unrestricted use, distribution, and reproduction in any medium, provided the original work is properly cited. Conditions, terms of use and publishing policy can be found at https://www.scienceopen.com/.

Preprint statement: This article is a preprint and has not been peer-reviewed, under consideration and submitted to ScienceOpen Preprints for open peer review.

DOI: 10.14293/S2199-1006.1.SOR-.PPOBUGW.v1

Preprint first posted online: 28 April 2020

Keywords: Superluminal velocity, The theory of relativity, Compressible ether, Superfluidity, Apparent physical quantity 


\title{
Superluminal velocity beyond the scope of application of relativity
}

\author{
Chang-Wei Hu \\ Beijing Relativity Theory Research Federation, Beijing, China \\ Shanghai Senior Scientist and technician Association, Shanghai, China
}

\begin{abstract}
People have carried on the extensive researches on the superluminal velocity in experiment and theory, but it is difficult to reach consensus. The biggest problem here is the theory of relativity, which shows that when a object (a matter with mass) reaches or exceeds the speed of light, whose relativistic factor will become infinite or imaginary numbers, so it is impossible to superluminal motion. In fact, although relativity is quite correct quantitative theory, but it has certain limitations. Relativistic effects are the vacuum effects, not the substantive effects. Relativistic physical quantities are only apparent physical quantities expressed through ether(physical vacuum). The substantive physical quantities of an objects are proper physical quantities, which will not vary with the velocity. Moreover the ether in superluminal velocity would lose superfluidity, and thus the superluminal velocity is beyond its scope of application of relativity. Therefore studying superluminal velocity need not scruple the restriction of relativity. Human superluminal activities will involve gravitational shielding, superluminal communication and other supertechnologies.
\end{abstract}

Keywords: Superluminal velocity, The theory of relativity, Compressible ether, Superfluidity, Apparent physical quantity

\section{Introduction}

For human beings, flying out of the solar system and carrying out astronavigation is a very distant hope, but it is also an ambition that must be realized. Because, according to astronomers' speculations, in about 1.6 billion years, the core of the sun will be hot enough to fuse the outer hydrogen, which will cause the radius of the sun to expand to 260 times and become a red giant, and will inevitably lead to the destruction of the earth. So to continue, humans should be prepared to flee the solar system.

But it's not easy to escape from the solar system. The nearest star to our solar system, Proxima Centauri, is 4.22 light-years away. The planet that can be suitable for human survival may be dozens of light years away. Therefore, the astro-ships must be able to travel faster than light to fly over the stars at the shortest possible time. This makes it possible to further solve a series of challenges, such as the power of the spacecraft and the livelihood of astronauts.

Since the 1970s, many signs of superluminal velocity have been found through astronomical observations[1, 2].

Later, design experiments using quantum tunneling effect and electric current etc., the possible phenomena of superluminal motion was realized and observed[2, 3].

People have also explored the theories of superluminal velocity. Some scientists tried to deal with the problem of superluminal velocity within the framework of relativity. There are also scholars who jump out of the framework of relativity to explore the theories of Superluminal velocity[3,5-7].

The above experiments and theoretical discussions of superluminal motion show that there is a certain possibility of superluminal velocity, but there is no scientific consensus yet. The main 
reason is that the correctness of relativity is difficult to shake, and when the speed $\mathrm{v}$ reaches or exceeds the speed of light, the relativistic factor $1 / \sqrt{1-\mathrm{v}^{2} / c^{2}}$ will be infinite or imaginary number, and will lead to time inversion, causality reversal. How to solve these problems thoroughly? The theory of compressibility ether[8,9] reveals the physical mechanisms and limitations of relativity, which is expected to remove obstacles to the superluminal study.

\section{Relativity and compressible ether}

Modern physics has confirmed that the vacuum is not void. Physical vacuum is an matter in vacuum state and infinite distribution. It was called ether by us. Ether is everywhere. It's ether that caused for all the anomalies in modern physics.

Lorentz transformation is the core of relativity. Einstein derived the Lorentz transformation based on the principle of relativity and the principle of the invariance of the speed of light. These principles are like axioms in geometry, and Einstein's relativity is a system of axioms. Axioms are the starting point or basis of logical reasoning, which can not be proved in this system, that is, relativity can not answer the question of why the speed of light is constant and so on, so it is difficult to explain the physical mechanism of relativity.

we do not need such preconditions as the invariance of the speed of light, and the lorentz transformation can be deduced by the method of fluid mechanics. This process contains the logic of matter of the substantive connection between things, and thus, the physical mechanism of relativity can be revealed[10]. The process is quite simple: in fluid mechanics, there is a transformation of compressible fluid into incompressible fluid, which is substituted into the galileo transformation that express the absolute space-time view, and Lorentz transformation that express relativistic space-time view can be obtained. In this process, the corresponding fluid is required to be an infinitely distributed superfluid, and its speed of sound is the speed of light in vacuum. Such a fluid can only be etheric. Light is the sound in ether, more precisely, the second sound in the superfluid ether.

The above derivation process shows that ether is a compressible fluid in absolute space-time view, that is, its density is variable; in relativistic space-time view, it is incompressible, that is, fluid with constant density, the speed of light is naturally constant. The route of sound in air of uneven density is curved. Light also bends in the gravitational field because the gravitational potential corresponds to the ether density. However, since people have not realized that ether is a fluid, and regards ether as a relativistic four-dimensional space continuum, the rate of change of ether density in absolute space-time is regarded as the curvature of four-dimensional space, so the relativistic space bends in the gravitational field.

The absolute space-time view is characterized by the invariance of the space-time standard, while the speed of light can vary with the ether density; the relativistic space-time view is characterized by the invariance of the speed of light, while its space-time standard can vary with the the ether density in the absolute space-time view. Therefore absolute space-time view and relativistic space-time view are two different kinds of space-time view. The former does not consider the role of ether, is pure, real space-time view. Because ether is everywhere, the absolute space-time view will have certain errors quantitatively; relativistic space-time view observes world through the ether, is a material space-time view, but it can be identical with experimental data, because the experimental process is bound to be subjected to the action of ether. So the relativistic space-time view, although distorted by ether, is a fairly accurate quantitative 
description. In the case of low speed and weak gravitational field, the effect of ether is like a flat light mirror, its action very little, and the absolute space-time view can be used to describe accurately enough; in the case of high speed and strong gravitational field, the effect of ether is like a concave or convex lenses,and thus the relativistic effects occur. In other words, the relativistic effect is the lens effect of ether, which can all be attributed to the density change effect of ether.

There is a correspondence between relativistic and absolute time-space views: relativistic unit length is proportional to the interval of adjacent particles of ether; relativistic unit time is proportional to the time interval that light passing through ether particles interval; gravitational field is ether density field; the absolute value of gravitational potential corresponds to ether density; gravitational field intensity corresponds to ether density gradient; mass corresponds to the increment of density of ether packet (relative to ground state); energy corresponds to the increment of pressure of ether packet (relative to ground state). where the ground state refers to the ether density in the vacuum where an object is located, and different ether densities are different ground states. Visibly, the unit length, unit time, mass, energy all can vary with the ground state.

In the absolute space-time view, the gravitational field is the ether density field, and each object is accompanied by the ether density wave packet. The universe is the sum of all the objects and their accompaniment ether in time-space.In relativistic space-time view, the universe is the entirety of uniform and curved space-time and objects.

The characteristics of relativistic physical quantities are further analyzed below.

\section{Relativistic apparent physical quantity}

The formula that unit length $d l$, unit time $d t$ and mass vary with the velocity in special relativity is:

$$
\begin{aligned}
& d l=d l_{0} \sqrt{1-v^{2} / c^{2}} \\
& d t=\frac{d t_{0}}{\sqrt{1-v^{2} / c^{2}}} \\
& m=\frac{m_{0}}{\sqrt{1-v^{2} / c^{2}}}
\end{aligned}
$$

Where it is proper physical quantity that the subscript is 0 .

From the expressions (1),(2),(3), it can be seen that if the velocity reaches the speed of light, the relativistic unit length in the direction of velocity will shrink to 0 ; the unit time is infinitely long, that is, slow to stop; and the mass will tend to infinity. If the speed exceeds the speed of light, they are all imaginary numbers. What does that mean?

From the previous section, we can see that any object is accompanied by an ether density wave packet, and its relativistic basic physical quantity, such as length, time, mass, energy, will vary with the ground state, that is, closely related to the ether density in the absolute space-time view. Figuratively speaking: an ether density wave pack of an object is like an air bag jacket. The relativistic physical quantity depends on the tight-loose extent of the air bag, so they are just the apparent physical quantity. The proper physical quantity in relativity is its intrinsic physical quantity. In fact, observing from a frame of reference at rest relative to a moving body, the mass 
and size of a rigid body will do not change. Therefore, it can be considered that when the object reaches the speed of light, the time stops, the mass tends to infinity, and so on, in fact, what it reflects is not the real change of time, mass, etc. but but rather a manifestation of the tendency of the ether density to infinity.

When an object moves in the air, the air on its way will be compressed and produce resistance. When the moving speed of an object is close to the sound speed, the air density in front of it (in the direction of motion) will be compressed and greatly increased, thus forming a sound barrier. Similarly, as an object moves in ether, its relativistic mass and energy, that is, the density and pressure of the ether in its ground state, ascend with the increase of speed. when the velocity approaches the speed of light, this ether density and pressure will tend to a maximum value and become a "light barrier ". The relativistic effect is the ether density change effect, that is, the vacuum effect. The object itself is not complete follow the relativistic formulae. The appearance of infinity or imaginary number in relativistic formula is a manifestation beyond the scope of application of relativistic formula. Therefore studying superluminal velocity need not scruple the restriction of relativity.

\section{Superluminal ether decoupled from relativity}

The potential function equation of compressible fluid is nonlinear. A hypothesis is made in the derivation of the Lorentz transformation of fluid mechanics: imagine an object moving with velocity $v$ in an infinite compressible fluid, which will cause disturbances in the fluid such as velocity (including direction, size) and density, pressure, etc; assuming that these disturbances are of the first order infinitesimal quantity, a linearized equation can be obtained. The Lorentz transformation is derived from this linearization equation. This assumption holds only if the fluid, the ether, is completely superfluid. Therefore, the relativistic formula is only applicable to the ether of superfluid.

As we all know, the superfluid has a certain critical velocity, critical density and critical pressure and so on. When a certain critical line is broken, the ether will lose the superfluidity, and the relativistic formula will no longer be effective. Obviously, if the velocity of an object reaches the speed of light, the surrounding ether will be excited into photons, and its critical line of velocity, density, etc. will be broken, so that the relativistic formula is no longer valid. In fact, Einstein himself said :" For the large field density and the material density, field equations and the field variables in these equations would not have the true meaning...... Overall, We need a clear understanding that the equations can not be extended to this region." [11]

To affirm the existence of superluminal velocity is not to deny relativity. In the scope of application, relativity is established, beyond the scope of application, relativity is no longer effective.

\section{Breakthrough of Light barrier}

The compressible ether theory holds that light is the "second sound" in the superfluid ether. In the past, the reason why people thought that the propagation of light did not need a medium was that in relativity, ether existed in the form of a four-dimensional space-time continuum and lost the image of "matter". In terms of compressible ether theory, superluminal velocity is comparable to the supersonic.

In fact, the supersonic research process has also experienced the dilemma of studying superluminal velocity now. Under the linearized theory of small perturbations in early subsonic flow studies, for necked pipe flow (see Figure 1, left), If the density of the fluid at rest is $\rho$, 
Then when the relative velocity is $v$, the fluid density $\rho^{\prime}$ is:

$$
\rho^{\prime}=\frac{\rho}{\sqrt{1-v^{2} / c^{2}}} \quad(v<c)
$$

Where the $c$ is the speed of sound. The formula (4) is identical to the formula (3). The resulting problem is comparable to the current superluminal velocity problem

The theoretical dilemma did not stop the pace of supersonic research. In the aspect of supersonic experiment, the early test is shown in figure 1 left, people want to continuously increase the pressure and reducing the cross section area of the outlet to increase the outlet velocity of flow. But it was found that the density (or energy) at the outlet also increased, and the velocity can never exceed the speed of sound. Later, an engineer named Rawal, who took a pipe with gradually enlarged section to connected behind the nozzle (Fig. 1 right), discovered that as long as the pressure was large enough, supersonic flow appeared in the enlarged section [12]! And in fact, when the speed reach the velocity of sound, the density of the gas increases only six times, not infinity [13].
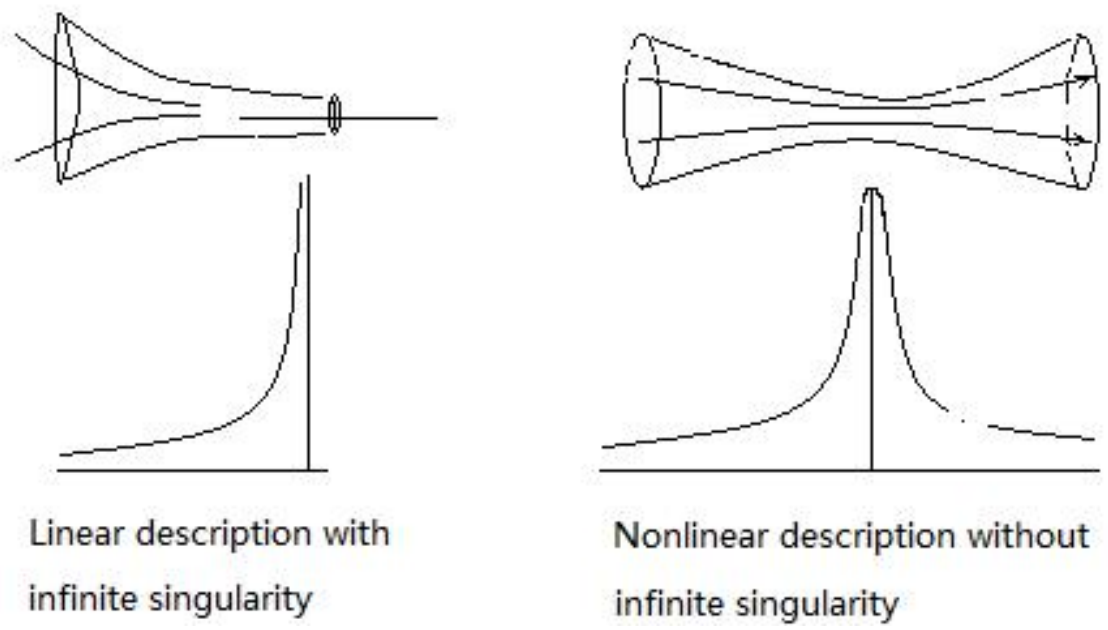

Figure 1 Supersonic discovery process

Human beings can break through the sound barrier, and also break through the light barrier.

\section{Superluminal astronavigation have a long way to go}

The era of superluminal astronavigation is bound to come, but the journey will be very difficult.

The speed of light is six orders of magnitude higher than the speed of sound in air, and reaching superluminal speed will be several orders of magnitude more difficult than supersonic. To realize superluminal astronavigation, we should not only develop special materials, but also develop a power system that can continue to work, but also improve the life security system of astronauts.

People take supersonic aircraft, the aircraft's shell to protect people from the harm of outside high-speed, high-pressure airflow. Likewise, astronauts must be protected from the harm of high-speed, high-pressure ether-flow. However, metal shell can block air, but not ether. According 
to "gravitational field is ether density field ", it seems that blocking ether should be a" gravitational shield ". What material, what technology can shield gravity? It may take centuries for humans to find the answer to this question.

When an object moves in the air, its disturbance propagates outward with the speed of sound. When the speed of motion reaches or exceeds the speed of sound, there must be a shock wave. The sound behind the shock wave can not catch up, and the sound in front of the shock wave is shattered by the shock wave, which prevents the reversal of cause and effect, and limits the use of sound waves to establish communication with the outside world. By the same token, the spaceship of superluminal velocity will not turn back time, and can not use electromagnetic waves to communicate with the outside world. So, how to maintain communication with the outside world in astronavigation? Quantum remote communication has been proposed [14]. we believe that ether is also hierarchical. Generally so-called ether, are gravitational field ether, which is macroscopic interval field ether. there will also be microscopic interval field ether, cosmoscopic interval field ether and so on $[15,16]$. In which, the speed of sound in the cosmoscopic interval field ethe should be much faster than the speed of light, and will one day be used by human beings.

In addition, the high-speed and high-pressure ether flow where the superluminal spacecraft arrives has been stimulated into a high-energy electromagnetic fluid, which will also have a certain impact on the spacecraft instruments, facilities, and astronauts.

There are many difficulties to be overcome on the way to astronavigation.

\section{References}

[1] Shenglin Cao, Theory of Relativity and Cosmology in Finsler's Space-Time [M]. Beijing: Beijing Normal University Press, 2001.

[2]、Zhixun Huang, Theory and Experiment of Superluminal Velocity Research [M], Beijing, Science Press, 2005

[3] Tsao chang, Research on the theory of space-time in physics, Shanghai Science and Technology Literature Publishing House, 2019

[4] Bilaniuk O M P, Deshpande V K and Sudarshan E C G. "Meta" relativity[J]. Am. J. Phys. , 1962,30:718-723

[5]、 F.R. Tanguerini, Introduction to General Relativity [M], Shanghai Science and Technology Press ,1963,8-11]

[6]、 Shu-sheng Tan, From Special Relativity to Standard Space-time Theory, Hunan Science and Technology Press, 2007

[7] Wen-Xiong Yang, Modern Newtonian Mechanics, Shanghai Jiao Tong University Press, 2011

[8] Chang-Wei Hu, Derivation of the relativistic equations from classical continuum mechanics on the basis of a macroscopic vacuum, Physics Essays, Volume 27: Pages 375-379, 2014.

[9]、 Chang-Wei Hu, The theory of compressibility ether, modern physics 2017, 7(4): $112-133$.

https://doi.org/10.12677/mp.2017.74013。

[10] Chang-Wei Hu, The logic structure deriving Lorentz transformation, ScienceOpen Preprints, 2019.11.13. ID: 10.14293/S2199-1006.1.SOR-.PPBEOMV.v1 
[11] A. Einstein, The Meaning of Relativity, Beijing, Science Press, 1966, pp. 84-85.

[12] Xintie Yang, et al, Breaking through the Light Barrier Drawing Experience From Fluid Mechanics, Baidu Library, 2010.

[13] Zhixun Huang, Possibility of the Massive Particles Moving by Faster-than-light, Journal of Communication University of China (Natural Science) Vol. 22, No.3, June 2015, 1-16.

[14] Zhixun Huang, Superluminal Communication Based on Quantum Nonlocality, Frontier Science, March 2016, 57-78.

[15]、 Chang-Wei Hu, The Confusion of Modern Cosmology and the New Model, ScienceOpen Preprints,2019.10.22. ID: 10.14293/S2199-1006.1.SOR-.PPTYNQB.v1

[16] Chang-Wei Hu, Probing Into Space-Time View, ScienceOpen Preprints, ID: $10.14293 /$ S2199-1006.1.SOR-.PP7LRGB.v1 